04

\title{
Первые эксперименты по контр-инжекции атомов высокой энергии на токамаке Глобус-М
}

\author{
(C) Н.Н. Бахарев, ${ }^{1}$ П.Р. Гончаров, ${ }^{2}$ В.К. Гусев, ${ }^{1}$ В.И. Давыденко, ${ }^{3}$ Г.В. Задвитский, ${ }^{4}$ Г.С. Курскиев, ${ }^{1}$ \\ А.Д. Мельник, ${ }^{1}$ В.Б. Минаев, ${ }^{1}$ М.И. Миронов, ${ }^{1}$ М.И. Патров, ${ }^{1}$ Ю.В. Петров, ${ }^{1}$ Н.В. Сахаров, ${ }^{1}$ \\ А.Д. Сладкомедова, ${ }^{1}$ А.Ю. Тельнова, ${ }^{1}$ С.Ю. Толстяков, ${ }^{1}$ Ф.В. Чернышев, ${ }^{1}$ П.Б. Щеголев \\ ${ }^{1}$ Физико-технический институт им. А.Ф. Иоффре РАН, \\ 194021 Санкт-Петербург, Россия \\ ${ }^{2}$ Санкт-Петербургский политехнический университет Петра Великого, \\ 194064 Санкт-Петербург, Россия \\ ${ }^{3}$ Институт ядерной фризики им. Г.И. Будкера СО РАН, \\ 630090 Новосибирск, Россия \\ ${ }^{4}$ 3IJL UMR 7198 CNRS - Université de Lorraine, BP 70239 \\ F-54506 Vandoeuvre-lés-Nancy, Cedex France \\ e-mail: bakharev@mail.ioffe.ru
}

(Поступило в Редакцию 28 марта 2017 г.)

Впервые на токамаке Глобус-М проведены эксперименты по контр-инжекции атомов высокой энергии. Получен режим улучшенного удержания без неустойчивостей, локализованных на периферии. В то же время при контр-инжекции не обнаружено значительного роста ионной температуры и энергосодержания плазмы, что объясняется высоким уровнем потерь быстрых ионов. Увеличение тока плазмы и расстояния плазма-стенка не привело к повышению эффективности нагрева плазмы с помощью нейтральной инжекции, как это происходило в экспериментах с ко-инжекцией.

DOI: 10.21883/JTF.2017.12.45203.2273

\section{Введение}

Инжекция атомов высокой энергии по направлению тока плазмы (ко-инжекция) широко применяется на современных токамаках из-за низких потерь вводимой мощности и эффективной генерации токов увлечения. Тем не менее нейтральная инжекция (НИ) навстречу току плазмы (контр-инжекция) также представляет интерес, поскольку может быть использована для получения режима улучшенного удержания (Н-моды) без неустойчивостей, локализованных на периферии (ELM - edge localized mode), приводящих к импульсной нагрузке на элементы внутренних конструкций токамака и вызывающих их быструю эрозию [1]. В частности, контр-инжекция позволяет получить так называемый спокойный режим улучшенного удержания (quiescent Н-моду) [2], в котором отсутствуют неустойчивости типа ELM, не происходит накопления примесей, а Н-фактор выше, чем при применении ко-инжекции. Исследования также показали, что контр-инжекция обладает рядом других преимуществ, таких как пониженный порог по плотности при переходе в Н-моду. На токамаке Туман-3М при использовании контр-инжекции нижний порог Н-моды по плотности составлял $0.5 \cdot 10^{19} \mathrm{~m}^{-3}$, что в 2.5 раза ниже, чем в омическом режиме и в режиме с ко-инжекцией [3]. Другим преимуществом применения контр-инжекции является то, что она позволяет создавать сильное вращение плазмы, подавляющее резистивную моду, стабилизируемую стенкой (resistive wall mode). Так, на токамаке MAST вращение плазмы, создаваемое с помощью контр-инжекции ато- мов, превосходило вращение, создаваемое с помощью ко-инжекции [4].

В то же время необходимо отметить, что контр-инжекция атомов высокой энергии на сферических токамаках мало исследована. В первую очередь это связано с тем, что в мире существовало всего четыре сферических токамака, оборудованных инжекторами атомов высокой энергии - NSTX, MAST, Глобус-M и START (установка разобрана). Эксперименты на токамаках START и MAST показали, что высокие потери вводимой мощности, характерные для НИ на сферических токамаках, значительно возрастают при применении контр-инжекции и достигают 97\% [5] и 50-80\% [6] соответственно. Если на токамаке START при применении контр-инжекции не было замечено существенного улучшения удержания плазмы по сравнению с омическим режимом, то на токамаке MAST удержание плазмы значительно улучшалось, а Н-фактор был в 2 раза выше, чем при ко-инжекции. В настоящей статье описаны результаты первых экспериментов по контр-инжекции на токамаке Глобус-М.

\section{Условия проведения эксперимента и используемые методы}

Эксперименты проводились на компактном сферическом токамаке Глобус-М [7] (большой радиус плазмы $\approx 0.36 \mathrm{~m}$, малый радиус $\approx 0.24 \mathrm{~m})$. Дейтериевая плазма удерживалась в диверторной конфигурации с нижней $X$-точкой. Тороидальное магнитное поле $B_{\text {tor }}$ составляло $0.4 \mathrm{~T}$, ток плазмы $I_{p}$ варьировался от 120 до $200 \mathrm{kA}$. 


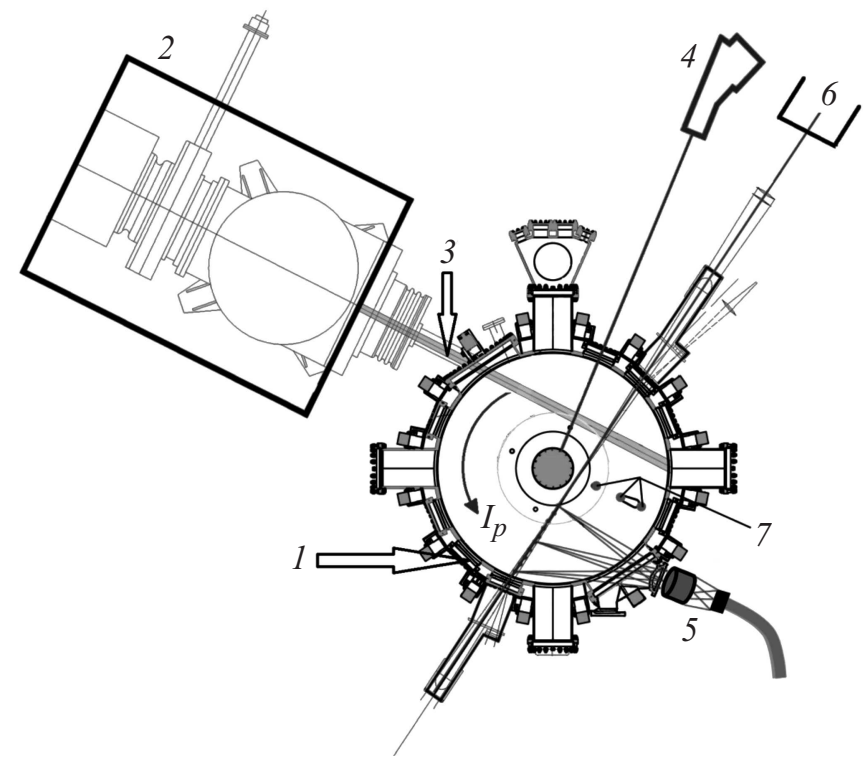

Рис. 1. Схема эксперимента. 1 - токамак Глобус-М, 2 - комплекс нейтральной инжекции, 3 - линия инжекции, 4 - анализатор атомов перезарядки АКОРД-12, 5 - объектив системы томсоновского рассеяния, 6 - лазер системы томсоновского рассеяния, 7 - каналы СВЧ интерферометра.

В качестве источника атомов высокой энергии использовался комплекс нейтральной инжекции [8]. В экспериментах применялся водородный пучок с энергией $18 \mathrm{keV}$ длительностью 30-40 ms, при этом вводимая мощность составляла 260-310 kW. Схема эксперимента показана на рис. 1. Инжекция пучка осуществлялась в направлении против тока плазмы. Профили электронной плотности и температуры измерялись с помощью диагностики томсоновского рассеяния лазерного излучения [9]. Значение среднехордовой электронной плотности определялось с помощью СВЧ интерферометра. Центральная ионная температура измерялась анализатором АКОРД-12 [10], линия наблюдения которого направлена вдоль большого радиуса токамака в экваториальной плоскости. Значение электронной плотности и температуры вблизи последней замкнутой магнитной поверхности измерялось с помощью подвижного ленгмюровского зонда. Магнитная конфигурация восстанавливалась с помощью кода EFIT [11], а профиль ионной температуры - с помощью кода DOUBLE [12]. Для расчета потерь быстрых частиц применялись коды NUBEAM [13] и трехмерный алгоритм, вычисляющий траектории частиц [14], объединенный с решением уравнения Больцмана [15]. Данные коды используют разные подходы для моделирования поведения быстрых ионов в токамаке, а их применение на токамаке Глобус-М было верифицировано в режиме ко-инжекции [16]. Для расчета орбит быстрых частиц в коде NUBEAM применяется обобщенное дрейфовое приближение, адаптированное для использования в сферических токамаках, а моделирование замедления быстрых ионов осуществляется с помощью статистического метода Монте-Карло.
Трехмерный алгоритм [14] вычисляет орбиты частиц без приближений путем решения уравнения движения в электрическом и магнитном полях. Данный метод требует больше компьютерных ресурсов, однако наиболее точно описывает траектории движения частиц. Замедление описывается кинетическим уравнением Больцмана со столкновительным членом Ландау [15] с учетом диффузии по скоростям и потерь на перезарядку.

\section{Влияние контр-инжекции на плазму токамака Глобус-М}

Удержание плазмы в сферическом токамаке Глобус-М в режиме без дополнительного нагрева и при ко-инжекции атомов высокой энергии достаточно хорошо изучено [16]. Режим улучшенного удержания (Н-мода), характеризуемый возникновением транспортного барьера для диффузии частиц, был получен как при использовании ко-инжекции атомов высокой энергии, так и за счет омического нагрева. Переход в Н-моду без дополнительного нагрева не приводил к увеличению энергетического времени удержания. При этом и в омическом режиме, и в режиме с ко-инжекцией атомов высокой энергии возникали неустойчивости, локализованные на границе (ELM).

Применение контр-инжекции атомов высокой энергии в токамаке Глобус-М дает возможность получения режима улучшенного удержания, без неустойчивостей, локализованных на границе (ELM-free H-моды). Это обстоятельство отличает исследуемый режим от омического режима и режима с ко-инжекцией высокоэнергетичных атомов, в которых существуют либо ELM III рода, характеризуемые высокой частотой повторения, либо ELM I рода, период которых совпадает с периодом пилообразных колебаний, либо и те, и другие. При контр-инжекции в ELM-free H-моде отсутствуют пилообразные колебания, но присутствует МГД неустойчивость с модой $m / n=2 / 1$, которая может подавляться пилообразными колебаниями в других режимах. На рис. 2 показаны характерные временные диаграммы мощности НИ, среднехордовой электронной плотности, интенсивности сигнала датчика $D \alpha$ в экваториальной плоскости и сигнала зонда Мирнова для разрядов с омическим нагревом дейтериевой плазмы, а также с ко- и контр-инжекцией водорода в дейтериевую плазму при токе плазмы $200 \mathrm{kA}$. После инициации перехода в режим улучшенного удержания во всех трех случаях, показанных на рис. 2, $a, b, c$, разряд находится в пограничном состоянии в течение 10-20 ms, совершая переходы между H-модой и L-модой (режим обычного удержания), после чего происходит окончательный переход в режим улучшенного удержания. Из рис. 2, с видно, что приблизительно через $5 \mathrm{~ms}$ после начала контр-инжекции разряд переходит в Н-моду без ELM, что отличается от режимов без инжекции или с инжекцией по направлению тока плазмы (рис. 2, $a, b)$. 

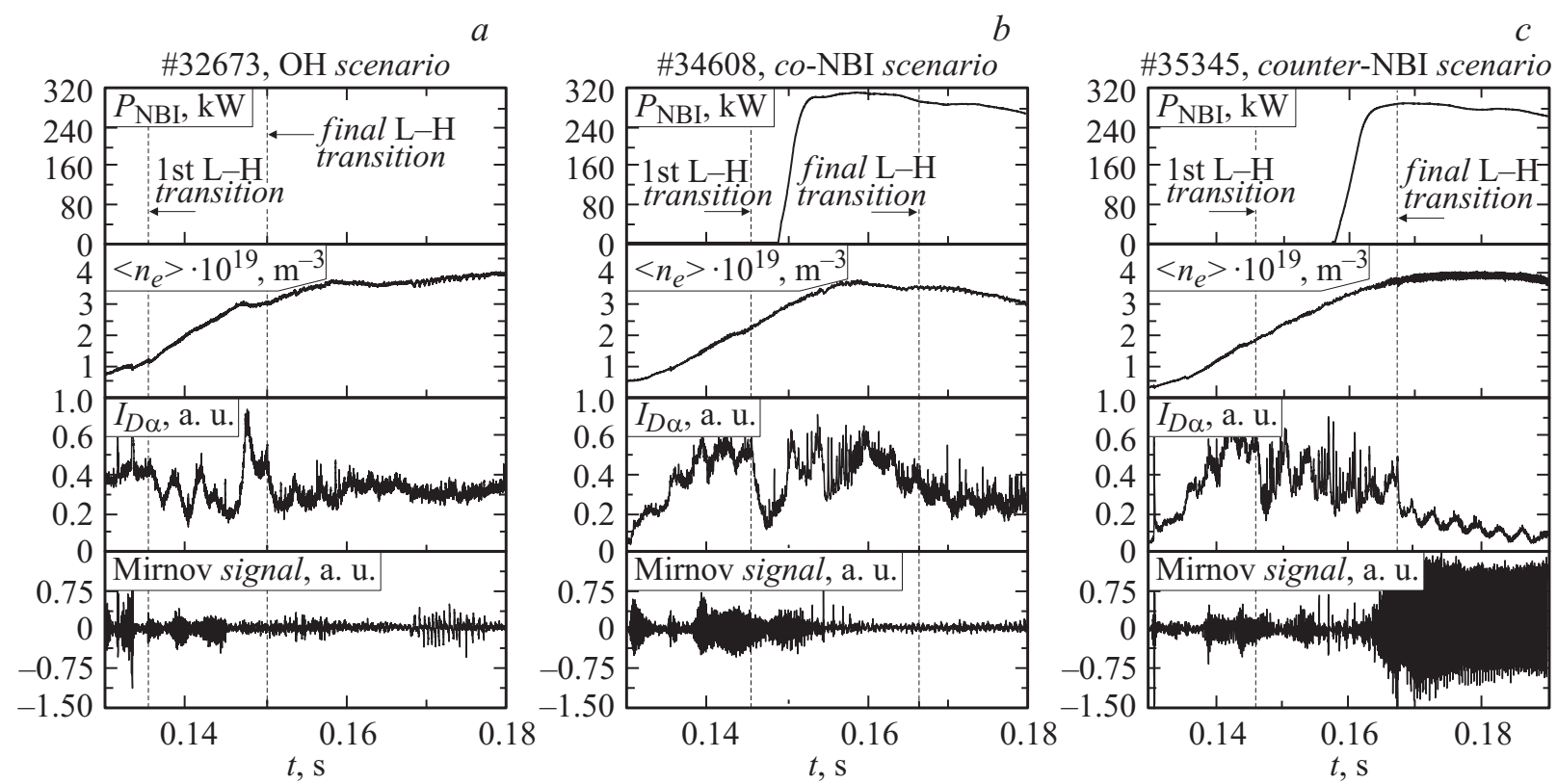

Рис. 2. Характерные временные диаграммы мощности НИ $\left(P_{\mathrm{NBI}}\right)$, среднехордовой электронной плотности $\left(\left\langle n_{e}\right\rangle\right)$, сигнал датчика $D \alpha$ в экваториальной плотности $\left(I_{D \alpha}\right)$ и сигнал зонда Мирнова (Mirnov signal) для разрядов с $a-$ омическим нагревом плазмы, $b-$ ко-инжекцией, $c-$ контр-инжекцией.

Тот факт, что режим, полученный в результате применения контр-инжекции, является именно Н-модой, подтверждается измерением профилей электронной плотности, полученных с помощью диагностики томсоновского рассеяния, показанных на рис. 3. Видно, что при переходе в режим улучшенного удержания наблюдается рост плотности в точке $56 \mathrm{~cm}$, наиболее близкой к последней замкнутой магнитной поверхности $(58 \mathrm{~cm})$. Это связано с образованием пьедестала плотности из-за образования транспортного барьера для диффузии частиц. Этот пьедестал сохраняется при переходе в Н-моду без ELM.

Возможной причиной перехода в режим улучшенного удержания является высокое значение электрического поля, связанное с большими потерями быстрых ионов, а отсутствие ELM может быть объяснено уменьшением градиента давления на границе из-за существования моды $m / n=2 / 1$ (рис. 2,c). Моделирование с помощью трехмерного алгоритма для расчета траекторий частиц показывает, что потери ионов с энергией $18 \mathrm{keV}$ с первой орбиты составляют 85-95\%, а полные потери достигают 97\%, в то время как при ко-инжекции с такими же параметрами пучка и плазмы потери с первой орбиты приблизительно равны 15\% [17]. Отметим, что моделирование контр-инжекции с помощью кода NUBEAM также показывает существенные значения потерь с первой орбиты: около $50 \%$, в то время как полные потери составляют $\approx 70 \%$. Занижение значений потерь в коде NUBEAM связано с использованием в нем дрейфового приближения. Из-за этого корректное применение NUBEAM возможно только для случая низких потерь ионов высокой энергии. В условиях, когда потери велики, как в случае с контр-инжекцией атомов высокой энергии, результаты расчетов с помощью кода NUBEAM можно использовать только для качественной оценки.

Высокие потери быстрых частиц также косвенно подтверждаются в эксперименте. Рост ионной температуры во время контр-инжекции не превышает 10\% по сравнению с омическим режимом, в то время как при ко-инжекции в тех же условиях достигается двукратное увеличение ионной температуры. Кроме того, в экспериментах

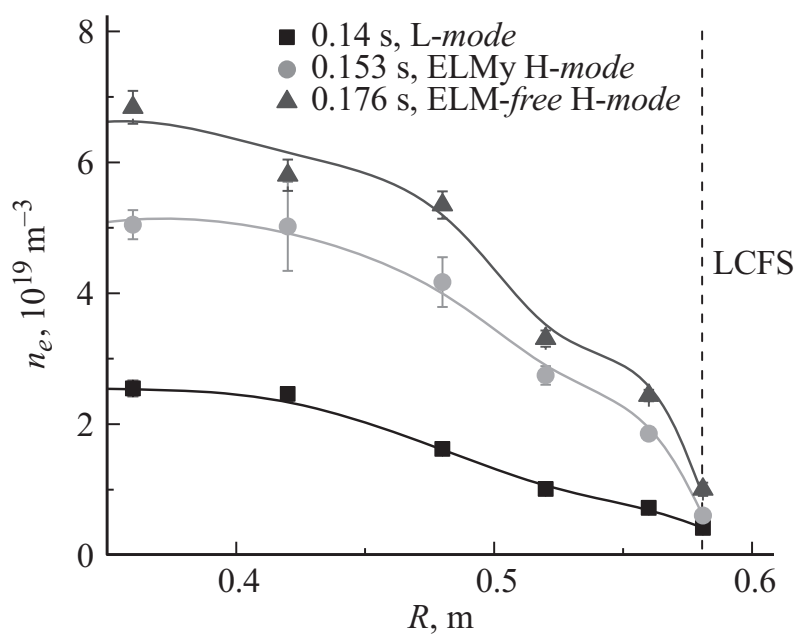

Рис. 3. Профили электронной плотности в разряде \#35345, полученные с помощью системы томсоновского рассеяния лазерного излучения. Штрихами показана последняя замкнутая магнитная поверхность. Значение плотности на последней замкнутой магнитной поверхности получено с помощью измерений подвижным ленгмюровским зондом. 


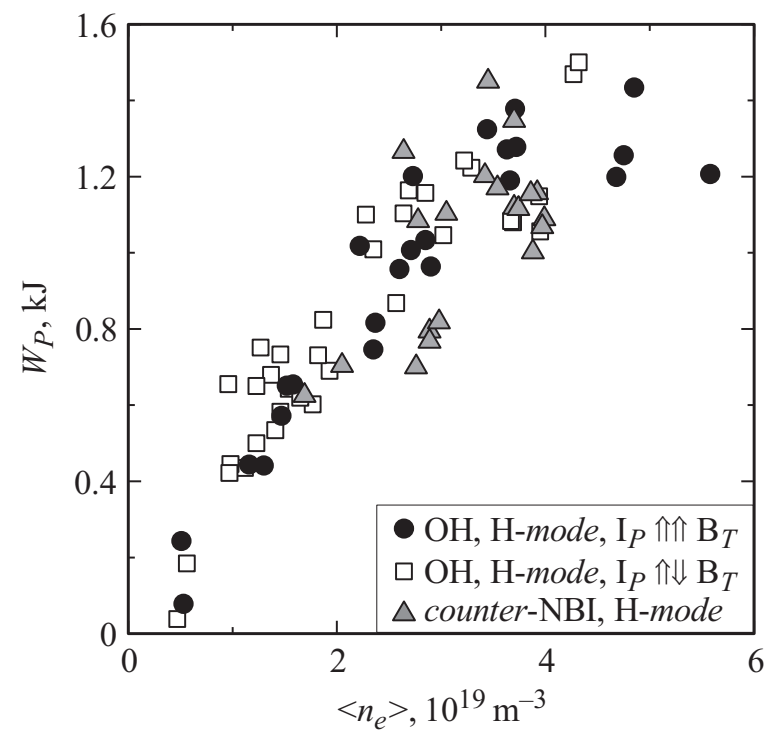

Рис. 4. Зависимость энергетического содержания плазмы токамака Глобус-М от среднехордовой электронной плотности плазмы для режима с омическим нагревом (случай, когда ток плазмы и тороидальное магнитное поле сонаправлены и разнонаправлены) и с контр-инжекцией атомов высокой энергии.

с ко-инжекцией уровень потерь быстрых частиц ниже, поэтому применение пучка атомов с энергией $26 \mathrm{keV}$ мощностью до $600 \mathrm{~kW}$ не приводит к срыву разряда, как это происходит в случае контр-инжекции. Благодаря этому при ко-инжекции легко удается получить рост ионной температуры более чем в три раза. Наличие высоких потерь быстрых частиц также подтверждается отсутствием изменения энергосодержания плазмы по сравнению с омической Н-модой, определяемого с помощью диамагнитных измерений. Это обстоятельство подтверждает рис. 4, на котором показана зависимость тепловой энергии плазмы токамака Глобус-М от ее среднехордовой электронной плотности в омической Н-моде (случай, когда ток плазмы и тороидальное магнитное поле сонаправлены и разнонаправлены) и с контр-инжекцией атомов высокой энергии.

Эксперименты по ко-инжекции показали, что при увеличении тока плазмы и расстояния плазма-стенка со стороны слабого магнитного поля наблюдается повышение эффективности нагрева ионного компонента плазмы, связанное с уменьшением потерь быстрых частиц [18]. В аналогичных экспериментах с контр-инжекцией ток плазмы менялся от 120 до $200 \mathrm{kA}$, а расстояние плазма-стенка - от 3 до $6 \mathrm{~cm}$. Увеличение расстояния между плазмой и стенкой при контр-инжекции не привело к росту температуры ионов. Увеличение тока плазмы приводило к росту ионной температуры на $10 \%$, в то время как в экспериментах с ко-инжекцией при таком же увеличении тока наблюдался рост температуры ионов до $35 \%$. Незначительный рост ионной температуры при увеличении тока плазмы в эксперимен- тах с контр-инжекцией почти не связан с улучшением удержания быстрых частиц, а объясняется в основном увеличением мощности омического нагрева. Описанные отличия в экспериментах с ко- и контр-инжекцией связаны с разным характером потерь быстрых ионов с первой орбиты. Характерные орбиты ионов водорода с энергией $18 \mathrm{keV}$, теряемых из-за столкновения со стенкой, показаны на рис. 5. Как видно из рисунка, в случае ко-инжекции частица теряется, попадая на внешнюю стенку токамака из-за большой ширины орбиты. Изменение расстояния плазма-стенка приводит к сдвигу орбиты внутрь установки, а увеличение тока плазмы к сужению орбиты. В результате удержание быстрых частиц и эффективность нагрева плазмы улучшаются. В случае контр-инжекции частица теряется из-за того, что ее орбита попадает в нижний купол установки. Сдвиг внутрь и сжатие орбиты не приводят к изменению ее формы для большинства ионов, и, следовательно, слабо влияют на удержание быстрых частиц.

В токамаке Глобус-М2 [19], сборка которого завершается в настоящее время, тороидальное магнитное поле будет увеличено до $1 \mathrm{~T}$, а ток плазмы - до $500 \mathrm{kA}$. Моделирование показывает, что при контр-инжекции, как и в случае с ко-инжекцией [17], одновременное изменение тороидального магнитного поля и тока плазмы в два с половиной раза приведет к уменьшению потерь быстрых частиц, более существенному, чем в описанных выше

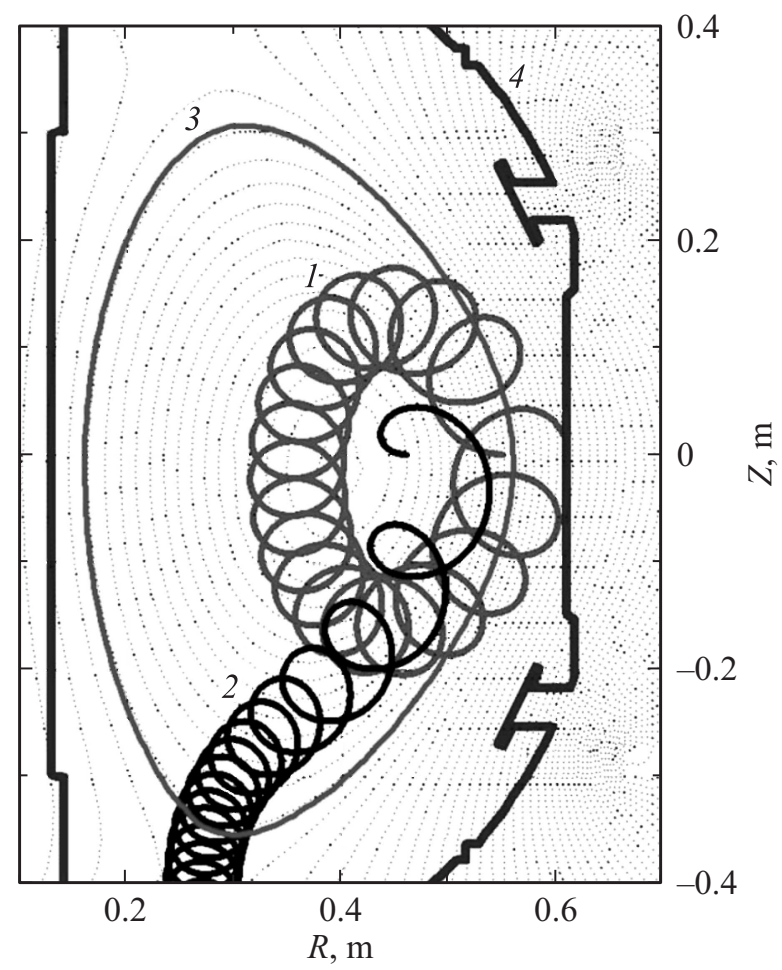

Рис. 5. Характерные орбиты теряемого из-за столкновения со стенкой иона водорода с энергией $18 \mathrm{keV}$ в полоидальном сечении токамака. 1 - случай ко-инжекции, $2-$ случай контр-инжекции, 3 - последняя замкнутая магнитная поверхность, $4-$ стенка вакуумной камеры токамака. 
экспериментах с изменением тока плазмы. Однако потери при контр-инжекции все еще будут велики и составят около 70\%. Улучшение удержания быстрых частиц позволит повысить энергию инжекции до $25-30 \mathrm{keV}$, в связи с чем мощность инжекции может быть поднята до 0.6-1 MW, что невозможно в описанных экспериментах из-за срыва разряда, вызванного повышенным поступлением примесей. На токамаке Глобус-М2 также планируется установка второго инжектора атомов высокой энергии с рабочими параметрами $50 \mathrm{keV}, 1 \mathrm{MW}$. Уменьшение прицельного параметра инжекции с 32 до $28 \mathrm{~cm}$ позволит снизить потери высокоэнергетичных ионов еще на 10\%. Несмотря на то что доля удерживаемых частиц будет мала, поглощенная мощность инжекции возрастет более чем на порядок по сравнению с токамаком Глобус-М и составит до $0.5 \mathrm{MW}$.

\section{Заключение}

Впервые на токамаке Глобус-М проведены эксперименты по контр-инжекции атомов высокой энергии. Получена ELM-free Н-мода. Отсутствие ELM может быть объяснено уменьшением градиента давления на границе из-за существования моды $m / n=2 / 1$, которая в разрядах без контр-инжекции подавляется пилообразными колебаниями. В токамаке Глобус-M ELM не представляют угрозы для элементов конструкции первой стенки и дивертора из-за низкого энергосодержания плазмы и, как следствие, низкой нагрузки на эти элементы. В более крупных установках будущего ELM необходимо ослабить или полностью подавить. Применение контр-инжекции для получения H-моды без ELM может быть удачной альтернативой методам контроля ELM благодаря своей простоте. В то же время улучшения удержания плазмы по сравнению с омической Н-модой в первых экспериментах по контр-инжекции на Глобус-М не зафиксировано, что может указывать на сходство результатов с установкой START.

Возможной причиной перехода в режим улучшенного удержания является высокое значение электрического поля, связанное с большими потерями быстрых ионов. Как показывают расчеты, полные потери быстрых частиц могут достигать 97\%. Высокие потери быстрых частиц косвенно подтверждаются экспериментально рост ионной температуры во время контр-инжекции не превышает $10 \%$. Также не наблюдается заметного роста энергосодержания плазмы. Изменение тока плазмы и расстояния плазма-стенка не приводило к улучшению удержания быстрых ионов, как это происходило в экспериментах с ко-инжекцией атомов высокой энергии. Это связано с различием орбит теряющихся ионов. При ко-инжекции частицы попадают на внешнюю стенку токамака, а при контр-инжекции - в диверторную область. Следует отметить, что в токамаках будущего нагрузка, создаваемая быстрыми ионами, теряемыми в диверторной области, представляет меньшую опасность, чем нагрузка, создаваемая быстрыми ионами, попадающими в стенку камеры. Тем не менее из-за того, что при контр-инжекции уровень потерь быстрых частиц выше, однозначный ответ об относительной опасности такой нагрузки можно дать только с помощью моделирования конкретных экспериментальных условий. Эксперименты по контр- инжекции будут продолжены на токамаке Глобус-М2. Моделирование предсказывает, что в новой установке поглощенная мощность инжектируемых атомов высокой энергии возрастет более, чем на порядок. Это, а также увеличение тороидального поля и тока плазмы позволит существенно расширить диапазон экспериментальных параметров.

Н.Н. Бахарев, Г.С. Курскиев, А.Д. Сладкомедова и П.Б. Щеголев благодарят за финансовую поддержку РФФИ, научный проект № 16-32-00027 мол_а.

\section{Список литературы}

[1] Federici G., Loarte A., Strohmayer G. // Plasma Phys. Control. Fusion. 2003. Vol. 45. P. 1523.

[2] Burrell K.H., Austin M.E., Brennan D.P. et al. // Plasma Phys. Control. Fusion. 2002. Vol. 44. P. 253-263.

[3] Lebedev S.V., Askinazi G.L., Chernyshev F.V. et al. // Nucl. Fusion. 2009. Vol. 49. P.85029.

[4] Helander P., Akers R.J., Eriksson L.G. // Phys. Plasmas. 2005. Vol. 12. P. 112503.

[5] Akers R.J., Appel L.C., Carolan P.G. et al. // Nucl. Fus. 2002. Vol. 42. P. $122-135$.

[6] Akers R.J., Helander P., Tournianski A.. et al. // Proc. 31st EPS Conf. on Plasma Phys., London, 2004. ECA Vol. 24G. P. $4.188 \mathrm{c}$.

[7] Gusev V.K., Bakharev N.N., Belyakov V.A. et al. // Nucl. Fus. 2015. Vol. 55. P. 104016.

[8] Гусев В.К., Деч А.В., Есипов Л.А. et al. // ЖТФ. 2007. Vol. 77. P. $28-43$.

[9] Курскиев Г.С., Толстяков С.Ю., Березуцкий А.А. и др. // BАНТ. Сер. Термоядерный синтез. 2012. Т. 2. С. 81-88.

[10] Izvozchikov A.B., Petrov M.P., Petrov S.Ya., Chernyshev F.V., Shystov I.V. // Tech. Phys. 1992. Vol. 37. P. 201.

[11] Lao L.L., John H. St., Stambaugh R.D., Kellman A.G., Pfeiffer W. // Nucl. Fus. 1985. Vol. 25. P. 1611.

[12] Afanasyev V.I., Gondhalekar A. and Kislyakov A.I. On the possibility of determining the radial profile of hydrogen isotope composition of JET plasmas, and of deducing radial transport of the isotope ions. 1999. JET-R-(00)04 www.iop.org/Jet/fulltext/JETR00004.pdf.

[13] Pankin A., McCune D., Andre R., Bateman G. and Kritz A. // Comput. Phys. Commun. 2004. Vol. 159. P. 157.

[14] Чернышев Ф.В., Абанасьев В.И., Гусев В.К. и др. // Физика плазмы. 2011. Т. 37. С. 595-615.

[15] Goncharov P.R., Kuteev B.V., Ozaki T., Sudo S. // Phys. Plasmas. 2010. Vol. 17. P. 112313.

[16] Kurskiev G.S., Gusev V.K., Sakharov N.V et al. // PPCF. 2017. Vol. 59. P. 045010.

[17] Bakharev N.N., Chernyshev F.V., Goncharov P.R. et al. // Nucl. Fus. 2015. Vol. 55. P. 55043023.

[18] Бахарев Н.Н., Гусев В.К., Ибляминова А.Д. и др. // Письма в ЖТФ. 2013. Т. 39. С. 22-30.

[19] Gusev V.K., Azizov E.A., Alekseev A.B. et al. // Nucl. Fus. 2013. Vol. 53. P. 093013. 